\title{
Effects of alcohol on pericardial adhesion formation in hypercholesterolemic swine
}

\author{
Antonio D. Lassaletta, MD, Louis M. Chu, MD, and Frank W. Sellke, MD
}

\begin{abstract}
Objective: Reoperative cardiac surgery is complicated in part because of extensive adhesions encountered during the second operation. The purpose of this study was to examine the effects of alcohol with and without resveratrol (red wine vs vodka) on postoperative pericardial adhesion formation in a porcine model of hypercholesterolemia and chronic myocardial ischemia.
\end{abstract}

Methods: Male Yorkshire swine were fed a high-cholesterol diet to simulate conditions of coronary artery disease followed by surgical placement of an ameroid constrictor to induce chronic ischemia. Postoperatively, control pigs continued their high-cholesterol diet alone, whereas the 2 experimental groups had diets supplemented with red wine or vodka. Seven weeks after ameroid placement, all animals underwent reoperative sternotomy.

Results: Compared with controls, pericardial adhesion grade was markedly reduced in the vodka group, whereas there was no difference in the wine group. Intramyocardial fibrosis was significantly reduced in the vodka group compared with controls. There was no difference in expression of proteins involved in focal adhesion formation between any groups (focal adhesion kinase, integrin alpha-5, integrin beta-1, paxillin, vinculin, protein tyrosine kinase 2, protein kinase $\mathrm{C} \varepsilon$, and phosphorylated protein kinase $\mathrm{C} \varepsilon$ ). The wine group exhibited elevated C-reactive protein levels versus the control and vodka groups.

Conclusions: Postoperative vodka consumption markedly reduced the formation of pericardial adhesions and intramyocardial fibrosis, whereas red wine had no effect. Analysis of protein expression did not reveal any obvious explanation for this phenomenon, suggesting a post-translational effect of alcohol on fibrous tissue deposition. The difference in adhesion formation in the vodka versus wine groups may be due to increased inflammation in the wine group. (J Thorac Cardiovasc Surg 2012;143:953-9)

Adhesion formation occurs in the mediastinum after cardiac surgical procedures and makes reoperative surgery particularly challenging, especially if the operation is performed within a few weeks of the initial surgery. These internal fibrinous attachments are thought to develop because of mesothelial trauma during an operation, which can result from surgical handling, instrumentation, exposure to air, temperature changes, surgical glove powder, and foreign bodies such as suture materials and implanted devices. ${ }^{1}$ Reduction in postoperative adhesion formation in cardiac surgery is a heavily researched topic by both the academic and the biomedical industry sectors. A significant reduction or elimination of adhesions could drastically decrease the difficulty of reoperative cardiac surgery by reducing operative time, reducing intra- and postoperative bleeding, improving patient

\footnotetext{
From the Department of Surgery, Division of Cardiothoracic Surgery, Warren Alpert School of Medicine, Brown University, Providence, RI.

Funding provided by National Heart, Lung, and Blood Institute Grants R01HL46716, R01HL69024, and R01HL85647 (to Dr Sellke), and National Institutes of Health Training Grants 5T32-HL094300 (to Dr Chu) and 5T32-HL076134 (to Dr Lassaletta). Disclosures: Authors have nothing to disclose with regard to commercial support. Received for publication Aug 31, 2011; revisions received Oct 24, 2011; accepted for publication Dec 13, 2011; available ahead of print Jan 13, 2012.

Address for reprints: Frank W. Sellke, MD, Division of Cardiothoracic Surgery, Cardiovascular Research Center, Warren Alpert School of Medicine, Brown University, 2 Dudley Street, MOC 360, Providence, RI 02905 (E-mail: fsellke@lifespan.org). $0022-5223 / \$ 36.00$

Copyright (C) 2012 by The American Association for Thoracic Surgery doi:10.1016/j.jtcvs.2011.12.016
}

outcomes, and even reducing operative costs. Small studies in animals have shown promising results in reducing adhesion formation with the use of bioabsorbable polymer films and barriers, ${ }^{2}$ topical application of growth factors, ${ }^{3}$ and fibrillar collagen membranes ${ }^{4}$ during the initial operation. However, large clinical trials are lacking or have failed to produce a reduction of postoperative adhesions in any clinically relevant fashion.

A substance that could be given perioperatively in a safe and cost-effective manner to significantly reduce the degree of adhesions seen at reoperation would have enormous impact potential in cardiac surgery. A recent study examining the nanoscale architecture of cell to extracellular matrix (ECM) adhesions using 3-dimensional super-resolution fluorescence microscopy delineated the protein interactions involved in adhesion formation. ${ }^{5}$ The interruption of these molecular interactions or inhibition of the deposition and cross-linking of collagen could reduce adhesion formation postoperatively. We hypothesized that the anti-inflammatory properties of resveratrol might modulate postoperative adhesion formation and designed an experiment to compare the effects of resveratrol-containing red wine with alcohol alone.

\section{MATERIALS AND METHODS \\ Animal Model}

Starting at 4 weeks of age, 28 male Yorkshire miniswine (Parson's Research, Amherst, Mass) were fed $500 \mathrm{~g} / \mathrm{d}$ of a high-cholesterol diet throughout 


$$
\begin{aligned}
& \text { Abbreviations and Acronyms } \\
& \begin{aligned}
\text { ANOVA } & =\text { analysis of variance } \\
\mathrm{CRP} & =\text { C-reactive protein } \\
\mathrm{ECM} & =\text { extracellular matrix } \\
\mathrm{FAK} & =\text { focal adhesion kinase } \\
\mathrm{HCC} & =\text { high-cholesterol control } \\
\mathrm{HCV} & =\text { high-cholesterol vodka } \\
\mathrm{HCW} & =\text { high-cholesterol wine } \\
\text { Int } \alpha 5 & =\text { integrin alpha-5 } \\
\mathrm{Int} \beta 1 & =\text { integrin beta-1 } \\
\mathrm{PKC} \varepsilon & =\text { protein kinase } \mathrm{C} \varepsilon \\
\text { p-PKC } \varepsilon & =\text { phosphorylated protein kinase } \mathrm{C} \varepsilon \\
\mathrm{PYK} 2 & =\text { protein tyrosine kinase } 2
\end{aligned}
\end{aligned}
$$

the 11-week experiment to simulate conditions of coronary artery disease. The hypercholesterolemic diet consisted of $4 \%$ cholesterol, $17.2 \%$ coconut oil, $2.3 \%$ corn oil, $1.5 \%$ sodium cholate, and $75 \%$ regular chow (Sinclair Research, Columbia, Mo). All animals had unlimited access to drinking water. After 4 weeks of diet modification, all animals underwent ameroid constrictor placement (Research Instruments SW, Escondito, Calif) to induce chronic cardiac ischemia. For all surgical procedures, anesthesia was induced with intramuscular injection of $5 \mathrm{mg} / \mathrm{kg}$ tiletamine $\mathrm{Hcl}$ (Telazol; Fort Dodge Animal Health, New York, NY). Animals were endotracheally intubated and mechanically ventilated at 12 to 20 breaths $/ \mathrm{min}$. Anesthesia was maintained with a gas mixture of 1.5 to $2.0 \mathrm{~L} / \mathrm{min}$ of $\mathrm{O}_{2}$ and $0.75 \%$ to $3.0 \%$ isofluorane. Surgical approach for ameroid constrictor placement was via a mini-left thoracotomy, and a titanium ameroid constrictor (1.75-2.25 mm internal diameter) was placed around the proximal left circumflex coronary artery just distal to its take-off from the left main coronary artery. The pericardium was reapproximated with 3 interrupted 4-0 Nurolon sutures (Ethicon, Somerville, NJ). Postoperative pain was controlled with buprenorphine hydrochloride (Buprenex; Ben Venue Laboratories, Inc, Bedford, Ohio) intramuscular injection (0.03 $\mathrm{mg} / \mathrm{kg}$ ) at the end of the first operation and placement of a transdermal fentanyl patch $(4 \mu \mathrm{g} / \mathrm{kg})$ continued for 72 hours postoperatively. All animals received perioperative aspirin at $325 \mathrm{mg} / \mathrm{d}$ for prophylaxis against thromboembolic events starting 1 day before the first operation and continuing for a total of 5 days.

Postoperatively the pigs were split into 3 groups according to diet supplementation. Pigs in the high-cholesterol control $(\mathrm{HCC})$ group $(\mathrm{n}=9)$ continued the high-cholesterol diet for the remaining 7 weeks of the experiment. The pigs in the high-cholesterol wine (HCW) group were supplemented with $375 \mathrm{~mL}$ of red wine (2009 Pinot Noir; Black Mountain Vineyard, Healdsburg, Calif) daily $(12.5 \%$ ethanol/volume, HCW, $\mathrm{n}=9)$. Pigs in the high-cholesterol vodka $(\mathrm{HCV})$ group were supplemented with $112 \mathrm{~mL}$ of vodka (Rubinoff Vodka, Somerville, Mass) daily (40\% ethanol/volume, HCV, $\mathrm{n}=9$ ).

Seven weeks after initial operation, at 15 weeks of age, all animals underwent a final nonsurvival operation via a median sternotomy. Pericardial adhesions were graded at the time of reoperation. Cardiac harvest included the collection of tissue from both the normal left ventricle, perfused by the left anterior descending artery, and the left ventricular tissue from the area at risk, in the distribution of the left circumflex coronary artery. Myocardial samples were immediately frozen in liquid nitrogen at the time of harvest or placed in a $10 \%$ formalin solution for paraffin embedment and tissue staining.

All experiments were approved by the Rhode Island Hospital Institutional Animal Care and Use Committees. Animals were cared for in accordance with the "Principles of Laboratory Animal Care" formulated by the National Society for Medical Research and the "Guide for the Care and Use of Laboratory Animals." 6

\section{Pericardial Adhesion Grading}

At the time of reoperation, after median sternotomy and exposure of the pericardium, epicardial to pericardial adhesions were evaluated using a grading system modified from a previously published porcine model. ${ }^{3}$ Difficulty in separating the pericardium from the epicardium was graded 0 to 3 as follows: $0=$ adhesions did not exist; $1=$ adhesions were filmy, light, with foamy dissection plane; 2 = adhesions were intermediate, requiring some sharp dissection, but mostly lysed by digital manipulation; and $3=$ adhesions were dense, easily bleeding, with marked obliteration of tissue plane, requiring exclusive sharp dissection. The overall adhesion rating for each pig was averaged into a mean for each group for comparison between groups.

\section{Measurement of Intramyocardial Fibrosis}

Paraffin sections of myocardial tissue from both the area at risk and normal left ventricle were prepared for trichrome staining. An area of myocardium free of vessels and representative of the entire sample was captured for each slide. Researchers were blinded to samples during staining, processing, and image analysis. Images were digitally captured at $10 \times$ magnification (Nikon Eclipse E800; Nikon Corporation, Tokyo, Japan) using a SPOT Camera (Diagnostic Instruments, Sterling Heights, Miss) and analyzed using Image J software (National Institutes of Health, Bethesda, Md) to calculate the percent of fibrosis (blue) of total myocardium (red). Numbers are reported in percent of myocardium replaced by collagen (fibrosis).

\section{Transmural Collagen Area Fraction}

Paraffin sections of transmural myocardial tissue from both the area at risk and the normal left ventricle were prepared for picrosirius red staining. Images were digitally captured at $20 \times$ magnification (Aperio ScanScope, Vista, Calif) and analyzed using Image $\mathbf{J}$ software to calculate the total transmural area in each sample, followed by measurement of the collagen area of the slide that stained red. Numbers are reported in collagen area fraction where collagen area fraction $=$ collagen area/transmural area.

\section{Protein Expression}

Sixty micrograms of total protein from the radioimmunoprecipitation assay buffer (Boston BioProducts, Ashland, Mass) soluble fraction of myocardial lysates made from the normal left ventricle tissue was fractionated by sodium dodecyl sulfate-polyacrylamide gel electrophoresis using the $\mathrm{Nu}$ Page Novex Bis-Tris Mini Gel system (Invitrogen, Carlsbad, Calif) and transferred to polyvinylidene fluoride membranes (Millipore, Bedford, Mass). Membranes were incubated with antibodies against focal adhesion kinase (FAK), integrin alpha-5 (Int $\alpha 5$ ), integrin beta- 1 (Int $\beta 1$ ), paxillin, vinculin, protein tyrosine kinase 2 (PYK2; Cell Signaling, Danvers, Mass), protein kinase $\mathrm{C} \varepsilon(\mathrm{PKC} \varepsilon)$, and phosphorylated protein kinase $\mathrm{C} \varepsilon$ (p-PKCE; Santa Cruz Biotechnology, Santa Cruz, Calif) at dilutions recommended by the manufacturer, followed by the appropriate horseradish peroxidase-linked secondary antibodies (1:2000; Jackson Immunoresearch, West Grove, Pa). Immune complexes were visualized via electrochemiluminescence and photographed using GeneSnap software (Syngene, Cambridge, England). Densiometry of electrochemiluminescence signal was performed using Image $\mathbf{J}$ software (National Institutes of Health). To ensure and correct for equal protein loading, membranes were probed with glyceraldehyde-3-phosphate dehydrogenase (Cell Signaling), a constitutively expressed housekeeping protein. Raw data collected as arbitrary light units from electrochemiluminescence fluorescence and Image $\mathbf{J}$ densitometry were averaged and expressed in fold change compared with HCC mean.

\section{Blood Draws and Serum Studies}

Blood was drawn under general anesthesia for serum analysis during the initial operation of ameroid placement and at the beginning of the reoperative sternotomy before the chest was opened. Three weeks after initial operation, animals were sedated with an intramuscular injection of $5 \mathrm{mg} / \mathrm{kg}$ 

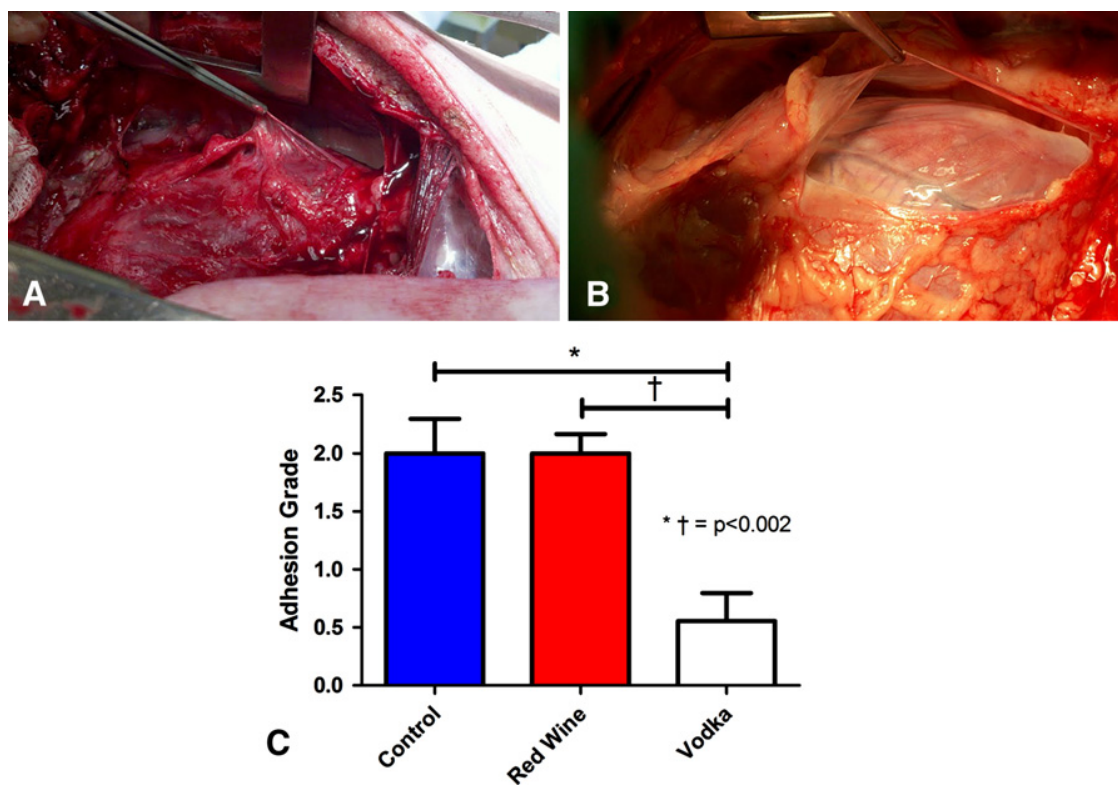

FIGURE 1. A, Pericardial adhesions observed 7 weeks after ameroid placement during reoperative sternotomy in HCC and HCW groups. B, Lack of pericardial adhesions observed in the HCV group. C, Pericardial adhesion grade is significantly reduced in HCV group compared with HCC and HCW groups.

tiletamine Hcl (Telazol) 1 hour after consumption of chow/supplement mixture, followed by percutaneous jugular venous blood sampling using anatomic landmarks. Serum was analyzed by the chemistry laboratory at the Rhode Island Hospital, Providence, Rhode Island.

\section{Statistical Analysis}

All results were reported as mean \pm standard error of the mean. Weight gain, adhesion grade, intramyocardial fibrosis, transmural collagen area fraction, molecular studies, and C-reactive protein (CRP) at the time of reoperation were analyzed by comparing means of each group using 1-way analysis of variance (ANOVA) followed by a post hoc Bonferroni test using GraphPad Prism 5.0 Software (GraphPad Software Inc, San Diego, Calif). Two-way ANOVA and Bonferroni post-tests were used to compare changes in CRP in each group from the first to second operation. The Student $t$ test was used for comparison between only 2 groups (blood alcohol levels between $\mathrm{HCW}$ and $\mathrm{HCV}$ ).

\section{RESULTS}

\section{Experimental Model}

There were no wound infections, dehiscence, or clinically different outcomes in wound healing in any of the animals. One animal from the HCW group died 9 days after ameroid placement. Necropsy did not reveal any obvious reason for death (which was likely due to ventricular arrhythmia), and the animal was excluded from analysis. Two animals from the HCC group died. One animal died after jugular venous blood draw, presumably from a tension pneumothorax, and the other animal died during the initial ameroid placement secondary to technical intraoperative complications. Both were excluded from analysis and replaced with new control animals.

\section{Weight Gain}

Weight gain from the first to second operations was not significantly different between the HCW and HCV groups
$(12.4 \pm 0.591 \mathrm{~kg}$ vs $11.7 \pm 0.281 \mathrm{~kg}, P=.249)$. However, there was significantly less weight gained in both the HCW and $\mathrm{HCV}$ groups compared with the controls $(16.5 \pm 1.505$ $\mathrm{kg}, P=.041$ and $P=.017$ for HCW and HCV vs HCC, respectively; ANOVA $P=.0063$ ).

\section{Pericardial Adhesions}

Mean adhesion grade at the time of the second operation (median sternotomy) was markedly reduced in the HCV group. By using the grading system described in "Materials and Methods," swine in the HCV group had significantly reduced adhesion scores compared with the HCC and HCW groups (ANOVA $P=.0003$, $\mathrm{HCC}$ vs $\mathrm{HCW} P=1, \mathrm{HCC}$ vs $\mathrm{HCV} P=.002$, HCV vs HCW $P=.0002$, Figure 1 ).

\section{Intramyocardial Fibrosis}

Trichrome staining of both the normal left ventricle and area at risk from each group showed grossly decreased intramyocardial collagen deposition in the HCV group. Statistical analysis using imaging software revealed significantly reduced intramyocardial fibrosis in the HCV group $(3.87 \% \pm 0.621 \%)$ compared with the HCC group $(10.32 \% \pm 2.19 \%, P=.002$, Figure 2$)$. There were marked differences in fibrosis in the HCW $(6.19 \% \pm 1.10 \%)$ group compared with the HCC group $(P=.081$, HCW $<$ HCC $)$ and $\mathrm{HCV}$ group $(P=.067, \mathrm{HCW}>\mathrm{HCV}$; ANOVA $P=.004)$, although they were not statistically significant.

\section{Transmural Collagen Area Fraction}

Picrosirius red staining of transmural myocardial sections showed significantly less collagen deposition in the $\mathrm{HCV}$ group compared with the HCC and HCW groups. 

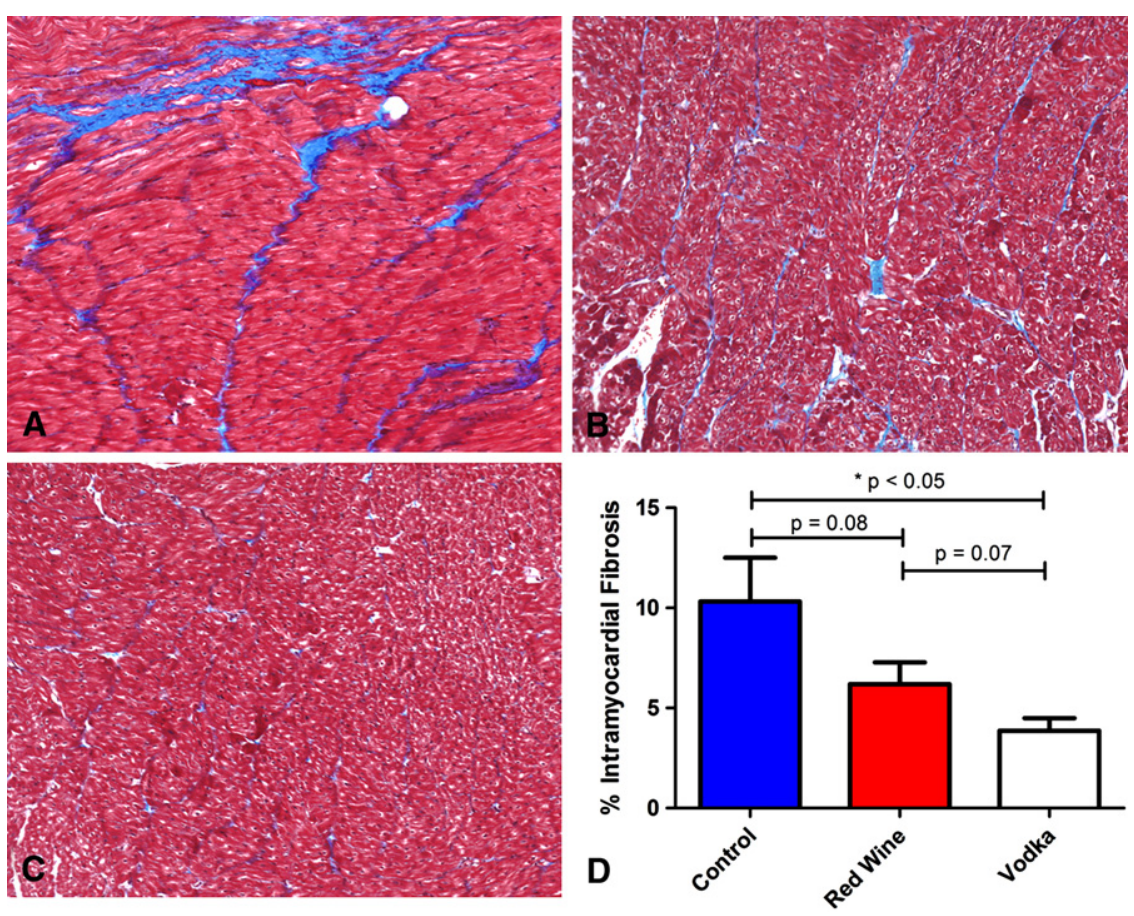

FIGURE 2. A, Trichrome staining showing intramyocardial collagen deposition (blue stain) replacing normal myocardium (red) of HCC group. Comparatively less collagen deposition is seen in the HCW group (B), and minimal collagen deposition is seen in the HCV group (C). D, Analysis of the percent of intramyocardial fibrosis is significantly reduced in the HCV group compared with the HCC group.

Collagen area fraction was $0.265 \pm 0.021$ in the HCC group compared with $0.189 \pm 0.025$ in the HCW group and 0.058 \pm 0.008 in the HCV group. One-way ANOVA analysis for all groups determined the $P$ value to be less than .0001 , and Bonferroni post-test determined all groups to be significantly different from each other (HCC vs HCW $P=.027$, HCC vs HCV $P<.0001$, HCW vs HCV $P<.0001)$.

\section{Protein Expression}

Western blot analysis of proteins involved in cell-ECM focal adhesion formation revealed no statistically significant differences in the expression of FAK, Int $\alpha 5$, Int $\beta 1$, paxillin, vinculin, $\mathrm{PYK} 2, \mathrm{PKC} \varepsilon$, or p-PKC $\varepsilon$ in the normal left ventricle (Figure 3).

\section{Blood Alcohol Levels}

The mean serum ethanol level was lower in the HCV group compared with the HCW group $(39.17 \pm 7.71$ $\mathrm{mg} / \mathrm{dL}$ in $\mathrm{HCW}$ vs $23.11 \pm 3.86 \mathrm{mg} / \mathrm{dL}$ in $\mathrm{HCV}, P=.06$, Figure 4). Blood was drawn from controls to verify that serum ethanol level was zero. A serum ethanol level of 40 $\mathrm{mg} / \mathrm{dL}$ correlates with a blood alcohol content of 0.04 on a standard breathalyzer.

\section{Markers of Inflammation}

Serum CRP levels were elevated in the HCW group (2.20 $\pm 0.425 \mathrm{mg} / \mathrm{L}$ ) at the time of reoperative sternotomy compared with the HCV and HCC groups $(1.32 \pm 0.142 \mathrm{mg} / \mathrm{L}$ and $1.48 \pm 0.154 \mathrm{mg} / \mathrm{L}$, respectively; ANOVA

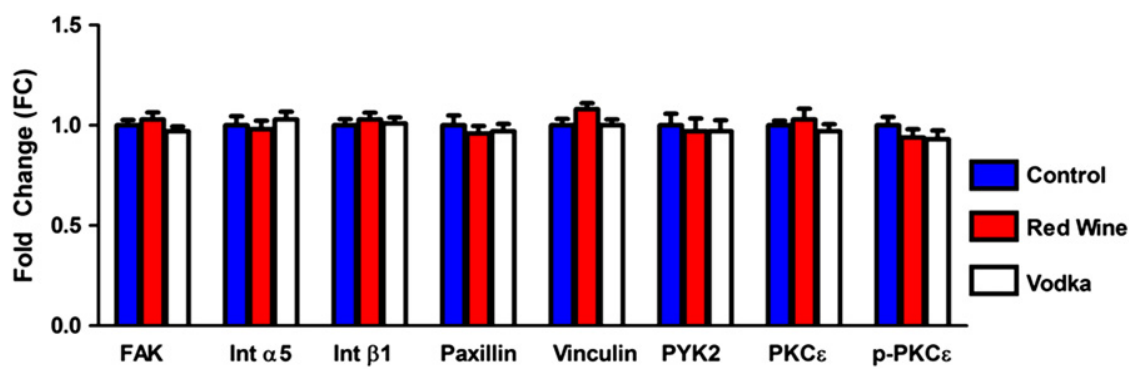

FIGURE 3. Expression of proteins involved in cell-ECM focal adhesion formation. There are no significant differences between groups. Levels represent fold change in mean compared with controls. $F C$, Fold change; $F A K$, focal adhesion kinase; Int $\alpha 5$, integrin alpha- 5 ; Int $\beta 1$, integrin beta-1; PYK2, protein tyrosine kinase $2 ; P K C \varepsilon$, protein kinase $\mathrm{C} \varepsilon ; p-P K C \varepsilon$, phosphorylated protein kinase $\mathrm{C} \varepsilon$. 


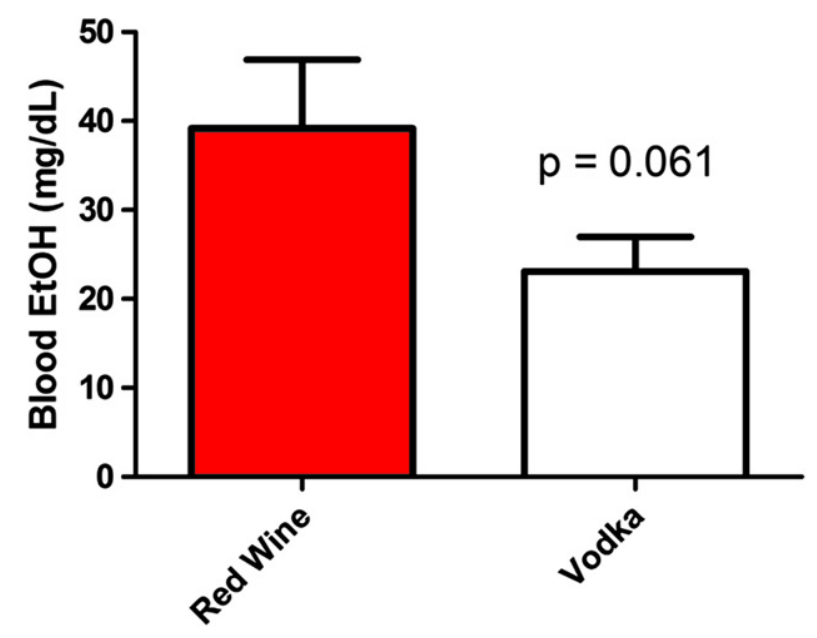

FIGURE 4. Blood alcohol levels are higher in the HCW group compared with the HCV group. EtOH, Ethanol.

$P=.0597)$. The change in serum CRP level from the first operation (pre) to reoperative sternotomy (post) was significantly different between groups (ANOVA $P=.0263$ ), with the largest difference between the HCW and HCV groups $(P<.05$, Figure 5). A trend toward similar changes in low-density lipoprotein cholesterol was observed between groups $(351,552$, and $410 \mathrm{mg} / \mathrm{dL}$ in the HCC, HCW, and HCV groups, respectively).

\section{DISCUSSION}

The current study tested the hypothesis that resveratrolcontaining red wine would reduce pericardial adhesion formation compared with alcohol alone. Resveratrol-free vodka was significantly more effective at reducing adhesions over the 7-week postoperative time period. This finding was supported by a statistically significant reduction in adhesion grade on a formalized scale. No difference was found in the expression of proteins known to be involved in focal adhesion formation among any groups (FAK, Int $\alpha 5$, Int $\beta 1$, paxillin, vinculin, $\mathrm{PYK} 2, \mathrm{PKC} \varepsilon, \mathrm{p}-\mathrm{PKC} \varepsilon$ ). One possible explanation for this negative finding in protein expression is that the mechanism of adhesion reduction is a post-translational modification effect on collagen deposition or cross-linking. Although there is overwhelming evidence that chronic alcohol abuse contributes to hepatic fibrosis, the effects of ethanol on cardiac and vascular tissues are not as well understood. Biomaterial research in the prevention of calcification and collagen deposition has shown ethanol pretreatment of porcine heart valves to be highly efficacious in preventing cuspal calcification and results in increased resistance to collagenase digestion. ${ }^{7}$ Furthermore, in rabbit studies, oral administration of perillyl alcohol led to a $22 \%$ reduction in intimal thickness of arterialized vein grafts. ${ }^{8}$ In our study, the differences in both intramyocardial fibrosis and transmural collagen deposition (controls $>$ red wine $>$ vodka) between groups support previous findings of the antifibrotic properties of ethanol in cardiac tissues and correlate with a reduction in adhesions. Another possibility is that there were differences in the expression of proteins involved in focal adhesion formation, but these differences are not apparent in the radioimmunoprecipitation assay soluble lysate fractions that were analyzed in this experiment. Because many of these cell-ECM adhesion proteins are at least in part transmembrane, it is possible that differences would be appreciated in analysis of the insoluble lysate fraction.

In our group's experience with several hundred ameroid constrictor placements via mini left thoracotomy followed by median sternotomy several weeks later, extensive, dense, hemorrhagic adhesions are universally encountered at the time of reoperation. The dense adhesions seen in this experiment enveloping all surfaces of the epicardium, not just the area involved in the mini-thoracotomy, in both the HCC and HCW groups support the assumption that the thoracotomy is a sufficient operation to create dense, easily bleeding pericardial adhesions.

All animals in the study were given a high-cholesterol diet to replicate as closely as possible the conditions present in humans with cardiac disease, the majority of whom have a degree of hypercholesterolemia and diabetes or glucose intolerance. Studies of surgically induced chronic ischemia in otherwise healthy animals have proven to be inadequate simulators of humans who have ischemic disease in the setting of multiple comorbidities. ${ }^{9}$ This lack of translatability
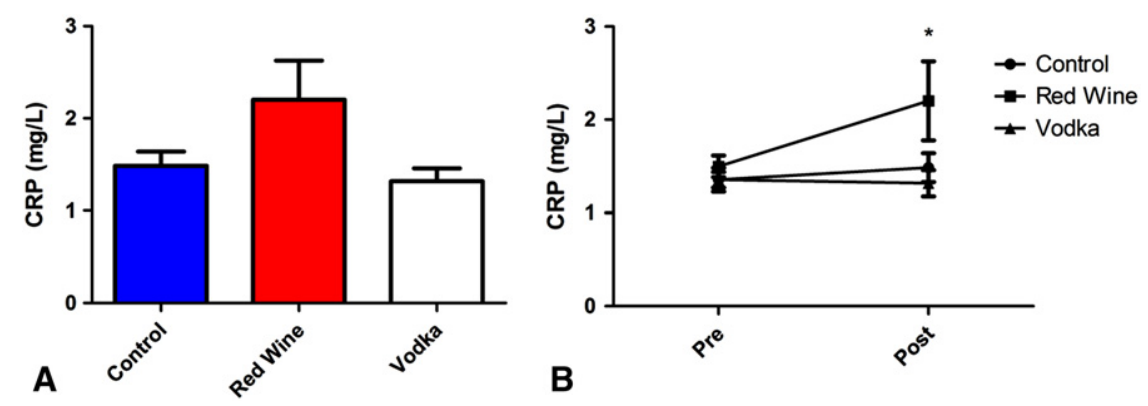

FIGURE 5. A, CRP levels are elevated in the HCW group at reoperation. $\mathrm{B}$, Changes in $\mathrm{CRP}$ are significantly higher $\left({ }^{*} P<.05\right)$ in the HCW group compared with the $\mathrm{HCV}$ and $\mathrm{HCC}$ groups. $C R P$, C-reactive protein. 
of large animal studies to clinical trials, although multifactorial, is due in large part to endothelial dysfunction that lacks in animal studies, yet is nearly omnipresent in humans with cardiac disease. Previous studies in swine have demonstrated that diet-induced hypercholesterolemia significantly alters endothelial function. ${ }^{10,11}$

Moderate alcohol consumption in humans has been shown to increase plasma levels of high-density lipoproteins, or "good cholesterol." Various lipoprotein functions are altered by alcohol intake, including the binding of high-density lipoprotein to cells, the modulation of growth factors, and the cytokine response. ${ }^{12}$ The effects of alcohol on inflammatory factors have not been as well delineated, because the effects of alcohol on CRP have yielded conflicting results ranging from different response curves depending on sex and body mass index ${ }^{13}$ to both proinflammatory ${ }^{14}$ and anti-inflammatory effects. ${ }^{15,16}$ According to the American Heart Association, CRP levels between 1.0 and $3.0 \mathrm{mg} / \mathrm{L}$ represent average risk for a cardiovascular event. ${ }^{17} \mathrm{~A}$ recent study in human alcohol consumption that stratified not only for increasing amounts of alcohol use but also for beverage type found wine consumption to be related to an increase in high-density lipoprotein, whereas beer and spirits were related to an increase in triglyceride levels. ${ }^{18}$ In this study, both CRP and lowdensity lipoprotein cholesterol levels were higher at reoperation in the animals that received red wine, and the change in CRP from the first operation to the second was significantly higher in this group, whereas no difference existed between the HCC and HCV groups. This elevated inflammatory state may explain why adhesions were seen in the $\mathrm{HCW}$ group versus the HCV group.

Because hypercholesterolemia increases markers of inflammation and oxidative stress, ${ }^{9}$ and alcohol consumption modifies lipoprotein function, ${ }^{12}$ as well as acting directly as an anti-inflammatory agent, ${ }^{16}$ it seems reasonable to deduce mechanisms by which vodka consumption decreases the inflammatory response: by modulating lipoprotein function in the setting of hypercholesterolemia or by directly decreasing lipoprotein concentration, thus decreasing the inflammatory response. Although this would be supported by the reduced adhesion formation seen in the HCV, one would also expect a reduction in adhesion formation seen in the pigs receiving an equivalent amount of alcohol from wine; this was not observed.

Resveratrol, which is found in the skin of red grapes, is thought to have several cardioprotective qualities due to its antioxidant, proangiogenic, and antiapoptotic properties. ${ }^{19}$ Why were adhesions not seen in the HCW group that was consuming the same volume of ethanol as the HCV group? Vodka is a simple mixture of ethanol and water, in contrast with red wine, which is a complex mixture of many compounds. Just as the resveratrol in red wine has been shown to have anti-inflammatory properties, it is possible that another compound in red wine inhibits the effects of ethanol on the post-translational modification of collagen deposition and cross-linking, if that is in fact the mechanism of adhesion reduction facilitated by vodka.

It is worth mentioning that despite the fact that the pigs were receiving identical volumes of alcohol in the HCW and HCV groups, the difference in serum ethanol levels between the groups was approximately a 2 -fold change in mean concentration (Figure 4). It is possible that there exists a "therapeutic window" for ethanol, as far as the inhibition of adhesion formation is concerned, and that the higher serum ethanol level in the wine group was outside this window. This explanation would be supported by observational studies in humans showing a U-shaped curve of CRP levels associated with alcohol consumption in which both nondrinkers and heavy drinkers had higher CRP concentrations than moderate drinkers. ${ }^{16}$ In fact, this U-shaped curve was observed in our study as well, with higher CRP levels in the nondrinkers (controls) and the group with the highest blood alcohol level (red wine) compared with pigs with a lower blood alcohol level (vodka). However, peak plasma concentrations for ethanol may vary as a function of rate of consumption or the concentration of alcohol in the substance being consumed. Thus, it is possible that the peak plasma concentration of ethanol occurred at different times depending on whether the animal received red wine or vodka.

The use of a systemic agent for adhesion reduction is not a novel idea. The benefits of localized treatment are obvious because systemic treatments carry the burden of undesired non-site-specific effects, such as with systemic administration of corticosteroids. However, as safe systemic agents are discovered and become available, current research is expanding into this realm. For example, recent studies in postoperative adhesion formation in rabbits have found systemic treatment with the monoclonal antibody sunitinib to significantly inhibit the formation of adhesions. ${ }^{20}$ The agents used in our study, red wine and vodka, have both been shown in the medical literature to be cardioprotective at moderate doses, supporting the safety of this agent for systemic administration. ${ }^{18,21,22}$ Finally, the blood levels of ethanol in this study $(\sim 20-40 \mathrm{mg} / \mathrm{dL})$ correlate with "moderate consumption" because the legal level of intoxication for ethanol in most US states is $80 \mathrm{mg} / \mathrm{dL}$. Thus, these levels are physiologically possible in humans.

\section{Limitations}

The most obvious limitation of this study is that of translatability of this swine model of hypercholesterolemia and chronic ischemia to a reduction in postoperative pericardial adhesions in humans. As mentioned previously, this model of ischemia with concomitant risk-factor modification is designed to better replicate the human disease state that it intends to model. It is also possible that one type of vodka or 
red wine may produce results different than were observed in the present study. Because there is yet no definitive mechanism for this observation of significant adhesion reduction, it would be premature to move into clinical trials. During reoperative sternotomy in patients, the mediastinum occasionally has minimal pericardial adhesions. A feasible next-step clinical study would be to identify differences in gene and protein expression in these patients to develop targets for novel therapeutic agents.

As with most large animal studies, because of the small numbers in the experiments, it is possible that despite statistically significant differences these findings may not be replicated in a larger study or clinical trial. Finally, a definitive mechanism will need to be established before this therapy will be acceptable after heart surgery.

\section{CONCLUSIONS}

The lack of a definite mechanism presents an obstacle for translation to clinical trials from this stage in animal studies, and a definitive or putative mechanism will likely need to be realized before this happens. However, this preliminary, large-animal study is pertinent to current clinical practice because these compounds are currently widely used by the general population, including patients undergoing cardiac surgery, and to ongoing research in both systemic and local treatments for postoperative adhesion reduction.

\section{References}

1. Holmdahl L, Risberg B, Beck DE, Burns JW, Chegini N, diZerega GS, et al. Adhesions: pathogenesis and prevention-panel discussion and summary. Eur J Surg Suppl. 1997;577:56-62.

2. Kaushal S, Patel SK, Goh SK, Sood A, Walker BL, Backer CL. A novel combination of bioresorbable polymeric film and expanded polytetrafluoroethylene provides a protective barrier and reduces adhesions. J Thorac Cardiovasc Surg. 2011;141:789-95.

3. Lopes JB, Dallan LA, Moreira LF, Campana Filho SP, Gutierrez PS, Lisboa LA, et al. Synergism between keratinocyte growth factor and carboxymethyl chitosan reduces pericardial adhesions. Ann Thorac Surg. 2010;90:566-72.

4. Bel A, Kachatryan L, Bruneval P, Peyrard S, Gagnieu C, Fabiani JN, et al. A new absorbable collagen membrane to reduce adhesions in cardiac surgery. Interact Cardiovasc Thorac Surg. 2010;10:213-6.
5. Kanchanawong P, Shtengel G, Pasapera AM, Ramko EB, Davidson MW, Hess HF, et al. Nanoscale architecture of integrin-based cell adhesions. Nature. 2010;468:580-4.

6. Clark JD, Gebhart GF, Gonder JC, Keeling ME, Kohn DF. Special Report: The 1996 Guide for the Care and Use of Laboratory Animals. ILAR J. 1997;38:41-8.

7. Vyavahare NR, Hirsch D, Lerner E, Baskin JZ, Zand R, Schoen FJ, et al. Prevention of calcification of glutaraldehyde-crosslinked porcine aortic cusps by ethanol preincubation: mechanistic studies of protein structure and water-biomaterial relationships. J Biomed Mater Res. 1998;40:577-85.

8. Schachner T. Pharmacologic inhibition of vein graft neointimal hyperplasia. J Thorac Cardiovasc Surg. 2006;131:1065-72.

9. Boodhwani M, Sellke FW. Therapeutic angiogenesis in diabetes and hypercholesterolemia: influence of oxidative stress. Antioxid Redox Signal. 2009;11: 1945-59.

10. Lassaletta AD, Chu LM, Sellke FW. Therapeutic neovascularization for coronary disease: current state and future prospects. Basic Res Cardiol. 2011;106:897-909.

11. Boodhwani M, Nakai Y, Mieno S, Voisine P, Bianchi C, Araujo EG, et al. Hypercholesterolemia impairs the myocardial angiogenic response in a swine model of chronic ischemia: role of endostatin and oxidative stress. Ann Thorac Surg. 2006; 81:634-41.

12. Brinton EA. Effects of ethanol intake on lipoproteins and atherosclerosis. Curr Opin Lipidol. 2010;21:346-51.

13. Oliveira A, Rodriguez-Artalejo F, Lopes C. Alcohol intake and systemic markers of inflammation-shape of the association according to sex and body mass index. Alcohol Alcohol. 2010;45:119-25.

14. Aziz-Seible RS, Lee SM, Kharbanda KK, McVicker BL, Casey CA. Ethano feeding potentiates the pro-inflammatory response of kupffer cells to cellular fibronectin. Alcohol Clin Exp Res. 2011;35:717-25.

15. Paulson QX, Hong J, Holcomb VB, Nunez NP. Effects of body weight and alcohol consumption on insulin sensitivity. Nutr J. 2010;9:14

16. Imhof A, Froehlich M, Brenner H, Boeing H, Pepys MB, Koenig W. Effect of alcohol consumption on systemic markers of inflammation. Lancet. 2001;357: 763-7.

17. American Heart Association. Inflammation, heart disease and stroke: the role of C-reactive protein. Available at: http://www.americanheart.org. Accessed June $15,2011$.

18. Foerster M, Marques-Vidal P, Gmel G, Daeppen JB, Cornuz J, Hayoz D, et al Alcohol drinking and cardiovascular risk in a population with high mean alcohol consumption. Am J Cardiol. 2009;103:361-8.

19. Wu JM, Hsieh TC. Resveratrol: a cardioprotective substance. Ann N Y Acad Sci. 2011;1215:16-21.

20. Meisel JA, Fallon EM, Le HD, Nehra D, de Meijer VE, Rodig SJ, et al Sunitinib inhibits postoperative adhesions in a rabbit model. Surgery. 2011; 150:32-8.

21. Hansel B, Thomas F, Pannier B, Bean K, Kontush A, Chapman MJ, et al. Relationship between alcohol intake, health and social status and cardiovascular risk factors in the Urban Paris-Ile-de-France Cohort: is the cardioprotective action of alcohol a myth? Eur J Clin Nutr. 2010;64:561-8.

22. Miyamae M, Kaneda K, Domae N, Figueredo VM. Cardioprotection by regular ethanol consumption: potential mechanisms and clinical application. Curr Drug Abuse Rev. 2010;3:39-48. 